\title{
An Architecture Framework for Measuring and Evaluating Packet-Switched Voice
}

\author{
Hyuncheol Kim ${ }^{1, \star}$, Seongjin Ahn ${ }^{2, \star \star}$, and Junkyun Choi ${ }^{1}$ \\ ${ }^{1}$ School of Engineering, Information and Communications University, \\ 119 Munjiro, Yuseong-Gu, Daejon, Korea, 350-714 \\ \{pharbor, jkchoi\}@icu.ac.kr \\ 2 Dept. of Computer Education, Sungkyunkwan University, \\ 53 Myungryun-Dong, Jongro-Gu, Seoul, Korea, 110-745 \\ sjahn@comedu.skku.ac.kr
}

\begin{abstract}
Until a recent date all telephony connections are set up via circuit switching. Advances in networking technology have made it possible for the Internet evolves into a Broadband convergence Network $(\mathrm{BcN})$ and provides various services including Internet Protocol (IP) telephony over high-speed IP networks. Voice-over-IP (VoIP) uses packetized transmission of speech over the Internet. In order for the Internet to realize a profit as traditional Public Switched Telephone Network (PSTN), it must provide high quality VoIP services. The VoIP metrics report block of Real-Time Transport Protocol Control Protocol Extended Reports (RTCP XR) can be applied to any one-to-one or one-to-many voice application for which the use of RTP and RTCP is specified. However, RTCP XR only defines packet type to convey information that supplements the six statistics that are contained in the report blocks used by RTCP's Sender Report (SR) and Receiver Report (RR) packets. Our objective in this paper is to describes a practical measuring framework for end-to-end QoS of packet switched voice in an IP environment including Packet Loss Concealment (PLC) techniques. It includes concepts as well as step-by-step procedures for setting up components, creating session, measuring packetized voice streams over IP networks.
\end{abstract}

\section{Introduction}

Until a recent date all telephony connections are set up via circuit switching. An alternate way of setting up end-to-end connections that is widely used for transmission of data is packet switching, such as that used in the Internet.

Advances in networking technology, digital media, and codecs have made it possible for the Internet evolves into a Broadband convergence Network $(\mathrm{BcN})$

\footnotetext{
* This work was supported by grant No. R01-2004-000-10618-0(2005) from the Basic Research Program of the Korea Science \& Engineering Foundation. This work was also supported in part by MIC, Korea under the ITRC program supervised by the IITA (IITA-2005-(ITAC1090050200070001000100100)).

$\star \star$ Corresponding author.
} 
and provides various services including Internet Protocol (IP) telephony and on-demand television over their high-speed IP networks.

Voice-over-IP (VoIP) uses packetized transmission of speech over the Internet (IP network) and has been thought as one of the killer application of $\mathrm{BcN}$. In order for the Internet to realize a profit as traditional Public Switched Telephone Network (PSTN), it must provide competent quality of services for VoIP systems comparable to traditional PSTN systems.

A large number of malicious factors are concerned to make a high-quality VoIP service. These factors include the speech codec, encoding (compression) schemes, packet loss, delay, delay variation, and the network architecture. Other factors involved in making a successful VoIP call includes the call setup signaling protocol, call admission control, security concerns, and the ability to traverse NAT (Network Address Translation) and firewall [1].

A successful end-to-end realization of IP telephony services presumes welldefined QoS measuring framework in the service provider's and customer's networks [2]. The VoIP metrics report block of Real-Time Transport Protocol Control Protocol Extended Reports (RTCP XR) can be applied to any oneto-one or one-to-many voice application for which the use of RTP and RTCP is specified. However, RTCP XR only defines packet type to convey information that supplements the six statistics that are contained in the report blocks used by RTCP's Sender Report (SR) and Receiver Report (RR) packets [3].

Our objective in this paper is to describes a practical measuring framework for end-to-end QoS of packet switched voice in an IP environment including Packet Loss Concealment (PLC) techniques and Network Time Protocol (NTP). It includes concepts as well as step-by-step procedures for setting up components, creating session, measuring packetized voice streams over IP networks. This paper also investigates the effects of packet loss and delay jitter on speech quality in VoIP scenarios.

The rest of this paper is organized as follows. In section 2, we will introduce a general components and control architecture of VoIP systems. The proposed VoIP QoS measurement architecture and functional components are described in section 3. In section 3, we will introduce their flow diagrams in detail so as to communicate with each other. we will also illustrate the performance of the system with extensive experimental data. Finally, Conclusions were drawn in Section 4

\section{VoIP Service and Components}

\subsection{VoIP Signaling Protocols}

As shown in Fig 1, Several standard protocols are available for building IP telephony solutions. These include H.323 from International Telecommunication Union - Telecommunication Standardization Sector (ITU-T); Media Gateway Control Protocol (MGCP), Session Initiation Protocol (SIP) from Internet Engineering Task Force (IETF); Media Gateway Control (Megaco) a joint protocol 


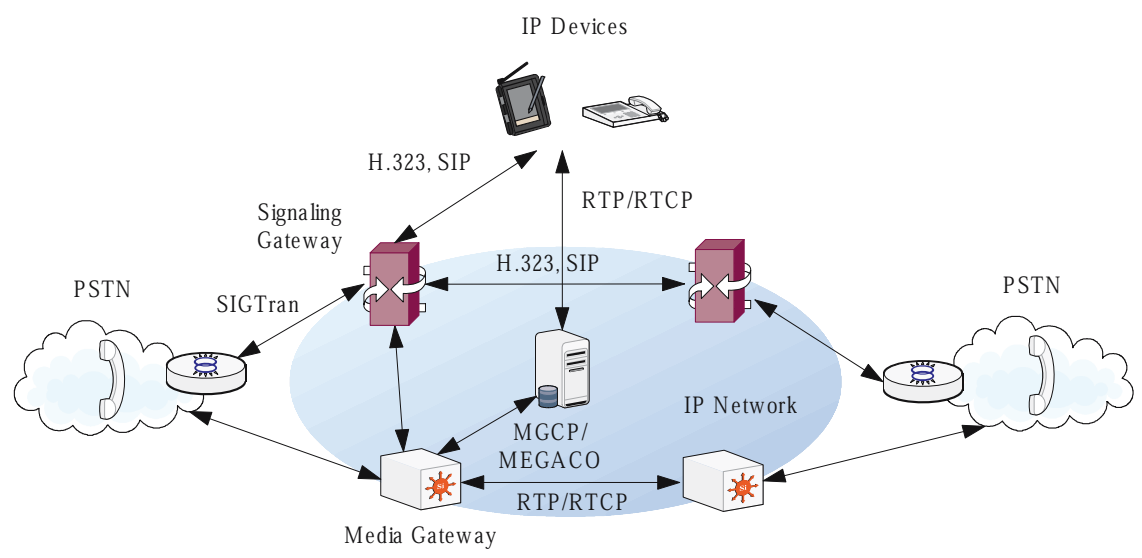

Fig. 1. Typical control plane architecture of VoIP system

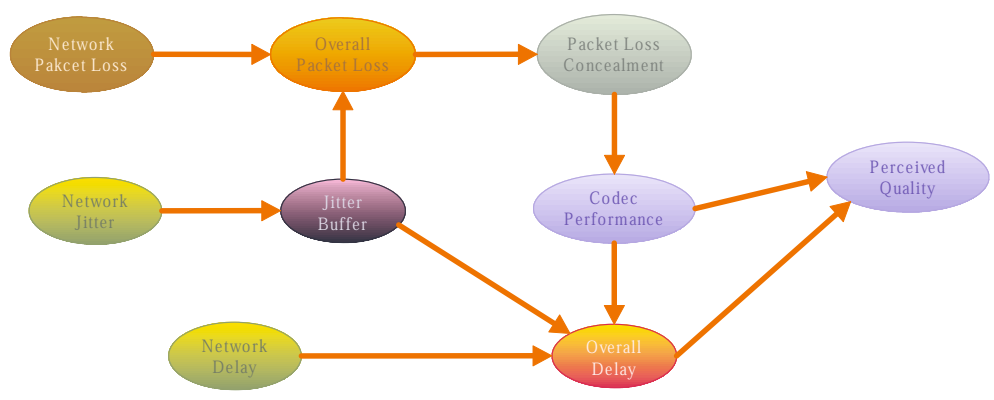

Fig. 2. VoIP quality of service parameter

by IETF and ITU-T; RTP and RTCP from IETF. The signaling protocols enable creating, modifying, and terminating multimedia sessions with one or more participants over IP networks. The responsibility for session establishment and signaling resides in the end stations.

SIP specifies procedures for telephony and multimedia conferencing over the Internet. SIP is an application-layer protocol independent of the underlying packet layer protocol (TCP, User Datagram Protocol (UDP), Asynchronous Transfer Mode (ATM), X.25). SIP is based on a client/server architecture in which the client (SIP User Agent (UA)) initiates the calls and the servers answer the calls. Because of its simplicity, scalability, modularity, and ease with which it integrates with other applications, SIP is attractive for use in packetized voice architectures [1] [4.

In order to provide inter-operability, a number of gateways provide for translation and call control functions between the two dissimilar network types. Encoding, protocol, and call control mappings occur in gateways between two 
endpoints. Along with signaling protocols, cooperation among various functions such as Call Admission Control (CAC), transcoding, interworking, and billing is essential to a successful realization of VoIP service [5].

\subsection{Quality of Voice}

Applications such as voice and video are particularly sensitive to network service quality. In VoIP applications a voice signal is first packetized and then transmitted over an IP network. However, at the receiving end, packets are missing or distorting due to network delay, network congestion (jitter) and network errors. This packet loss or delay degrades the quality of speech at the receiving end.

In order to estimate the quality of voice stream in the middle of session, it is essential to produce generalized quantitative measures that reflect the objective rating of the voice stream. The Mean Opinion Score (MOS) test is widely accepted as a standard for speech quality rating. However, the subjective MOS rating is time-consuming and inaccurate. In recent years, several objective MOS measures were developed, such as Perceptual Analysis Measurement System (PAMS) and Perceptual Evaluation of Speech Quality (PESQ).

The E-model standards, the E-model started as a research by European Telecommunications Standards Institute (ETSI), also provide a formula for calculating the loss of interactivity as function of the one-way delay. The E-model expresses an overall rating of the quality of a call and can be translated into quality and MOS 6] 7] 9$]$.

$$
R=\left(R_{0}-I_{s}\right)-I_{d}-I_{e}+A
$$

In equation (11), $R_{0}$ represents the basic signal-to-noise ratio, including noise sources such as circuit noise and room noise. The factor $I_{s}$ is a combination of all impairments which occur more or less simultaneously with the voice signal , such as side-tone and Pulse Code Modulation (PCM) quantizing distortion. Factor $I_{d}$ represents the impairments caused by delay and the equipment impairment factor and $I_{e}$ represents distortion of the speech signal due to encoding and packet loss. The advantage factor $A$ allows for compensation of impairment factors when there are other advantages of access to the user, e.g., when using cellular or satellite phone [8] 9].

E-Model is regarded as prominent rating technology that can be applied most properly when estimate speech quality for VoIP service because it considering about data network characteristic such as loss, delay.

\subsection{VoIP Transport Protocols}

The chief requirement that real-time media places on the transport protocol is for predictable variation in network transit time. RTP provides end-to-end network transport functions suitable for applications transmitting real-time data such as audio or video stream. 
RTP selects UDP as a transport layer because it has lower delay than TCP and because voice stream tolerates low levels of loss and cannot effectively exploit retransmission. RTP does not address resource reservation and does not guarantee QoS for real-time services.

The data transport is completed by a control protocol (RTCP) to allow monitoring of the data delivery and to provide minimal control and identification functionality. RTCP provides for reliable information transfer once the audio stream has been established.

RTCP provides feedback on the quality of data distribution and carries a transport-level identifier for an RTP source used by receivers to synchronize audio and video.

\section{Measurement Framework of Voice Stream in Packet Network}

In this section, we describe the architecture of the proposed VoIP measuring framework. The framework can be integrated to the commercial VoIP system as shown in Fig. 3 ,

The RTCP XR packets are useful across multiple applications, in particular, the VoIP metrics report block provides useful metrics for monitoring voice over IP (VoIP) calls. These metrics include packet loss and discard metrics, delay metrics, analog metrics, and voice quality metrics. However, little more detailed procedure need to be defined for real operation.

If a caller want to setup a session, as shown in Fig. 3. it will send INVITE message to callee to join the session. At the same time, as shown in Fig. 5(a). the caller will send type-1 initiation message to measurement server. After send

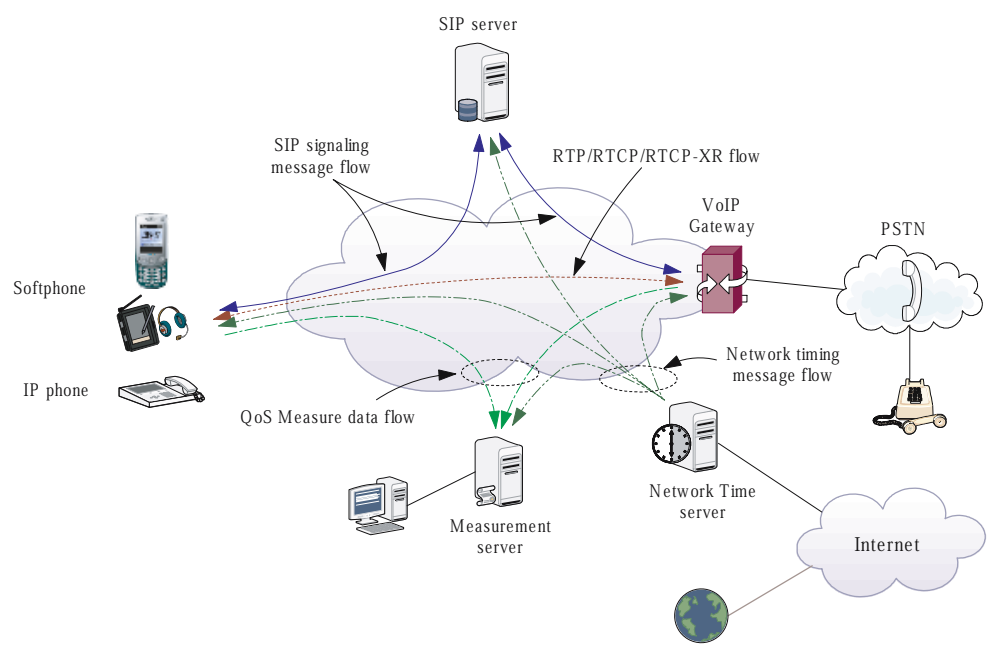

Fig. 3. VoIP measurement framework 


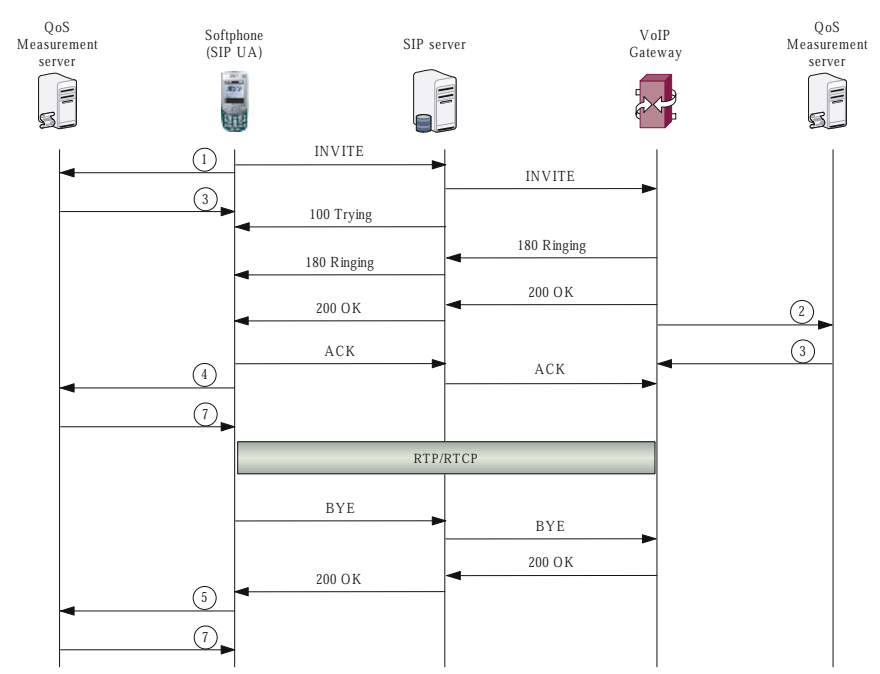

Fig. 4. VoIP QoS measurement procedure - normal session

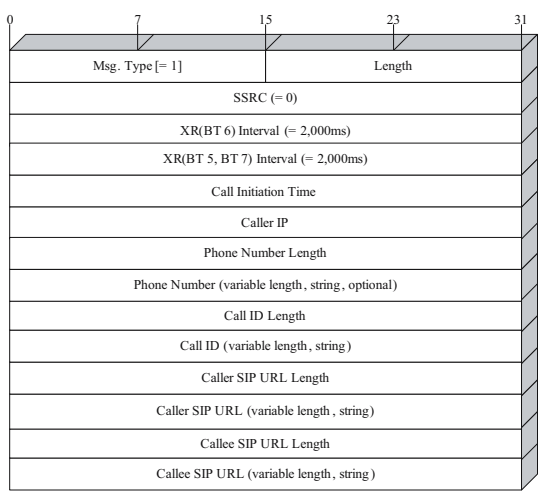

(a) Type-1 message

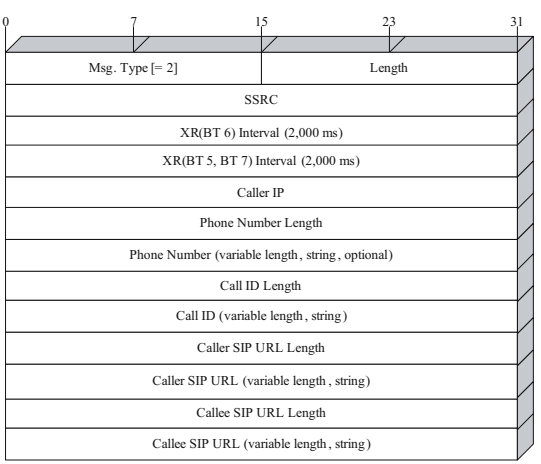

(b) Type-2 message

Fig. 5. Revised RTCP-XR initiation messages

200 OK message to the caller, as shown in Fig. 5(b), the callee will send send type- 2 initiation message to the measurement server. The measurement server will respond the initiation messages respectively.

When the caller receives the callee's respond, it will send ACK message to reply the respond. Immediately after that, the caller will also send type- 4 start message to the measurement server. After that the caller will setup the media channels such as RTP streaming with the caller.

If the caller or the callee do not want to join the session anymore, they will send BYE message to the other participant. After that, the caller and the callee will send type-5 end message to the measurement server. 


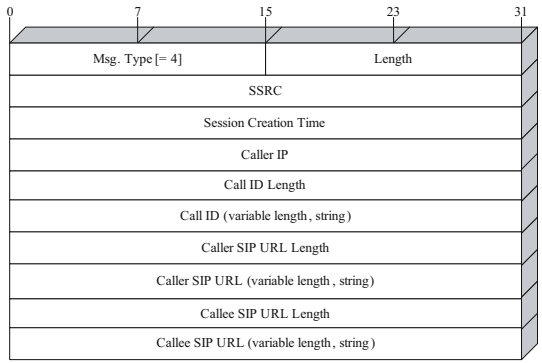

(a) Type-4 start message

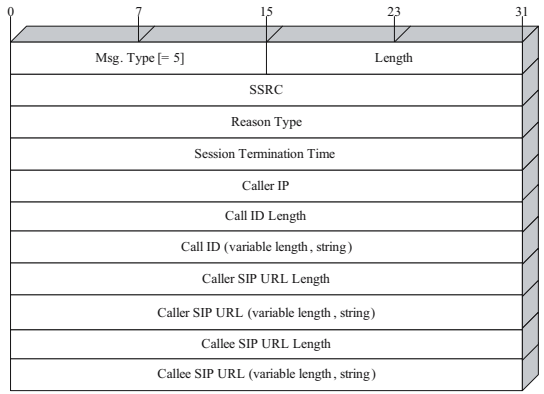

(b) Type-5 end message

Fig. 6. Revised RTCP-XR measuring start and end messages

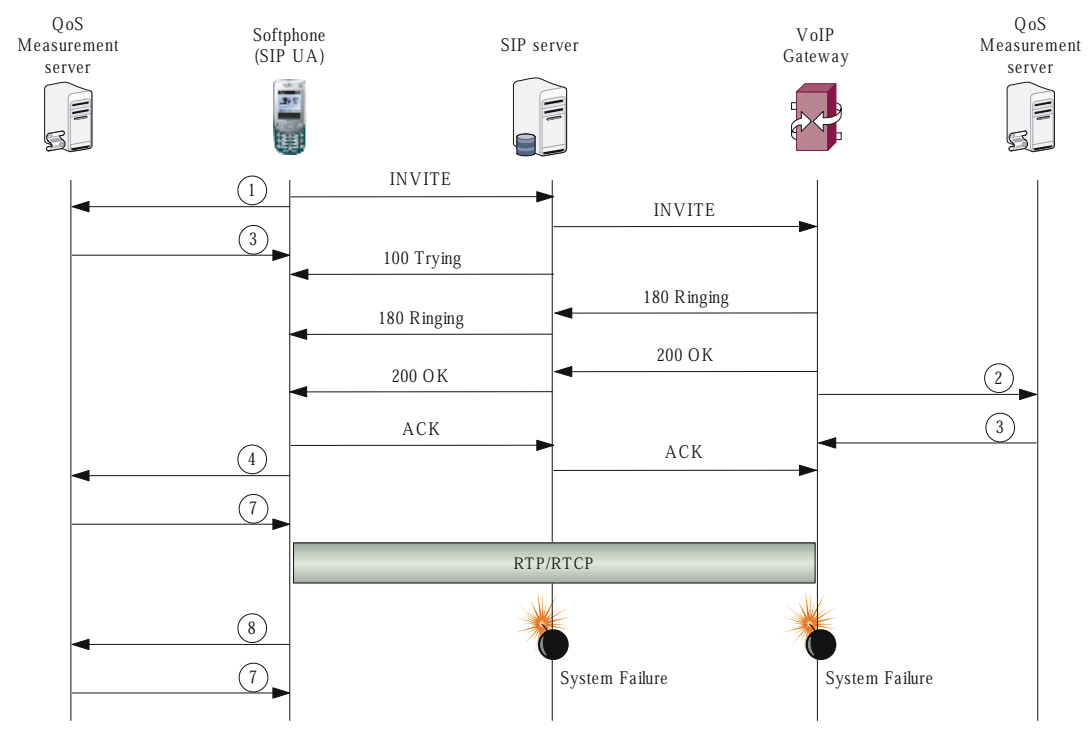

Fig. 7. VoIP QoS measurement procedure - an abnormal session

If the caller or the callee of the session can not setting up or lasting a session, as shown in Fig. 7 and Fig. 8, they will send type- 8 event message to the other participant. Fig. 9 shows the details of type- 8 event message.

The content of the received voice packets is delivered to the decoder, which reconstructs the speech signal. Decoders may implement Packet Loss Concealment (PLC) methods that produce replacement for lost data packets. Simple PLC schemes simply insert silence, noise, or a previously received packet. More sophisticated schemes attempt to find a suitable replacement based on the characteristics of the speech signal in the neighborhood of the lost packet(s). 


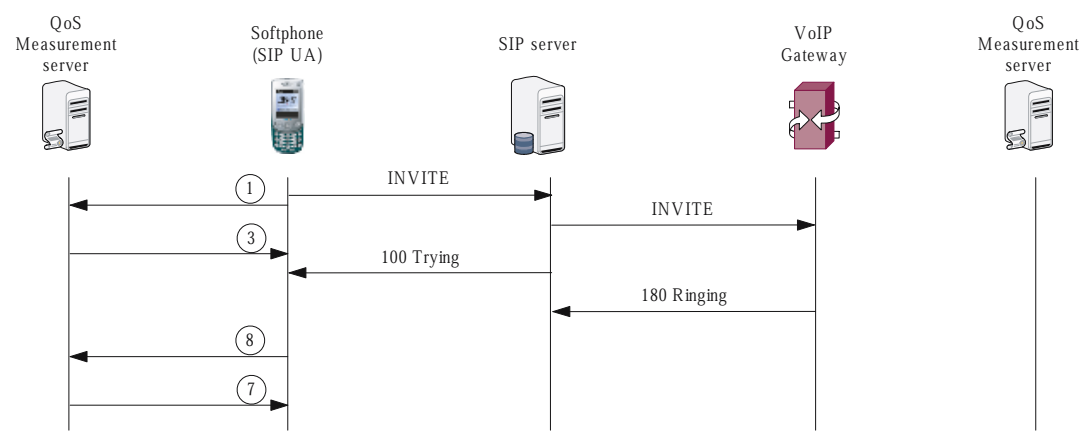

Fig. 8. VoIP QoS measurement procedure - a rejected session

\begin{tabular}{|c|}
\hline Msg. Type [- 8] \\
\hline SubType [2] \\
\hline Sender Count \\
\hline Receiver Count \\
\hline Option \\
$\cdots$ \\
$\cdots$ \\
\hline$\ldots$ \\
\hline
\end{tabular}

Fig. 9. Revised RTCP-XR Type- 8 event messages

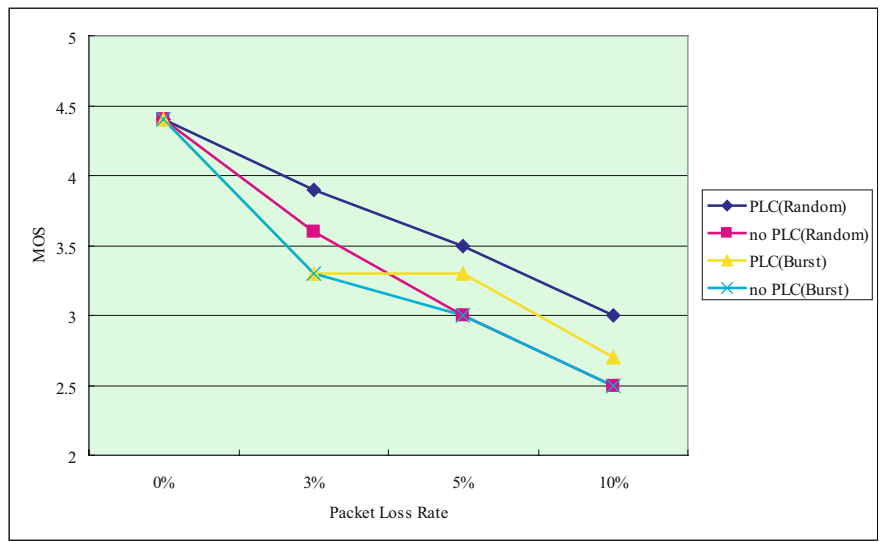

Fig. 10. Packet loss rate and MOS

Fig. 10 shows the relationship between (random/burst) packet loss and MOS value that is captured at the measurement server. Fig. 11] shows the relationship between (random/burst) packet loss and $\mathrm{R}$ rate. 


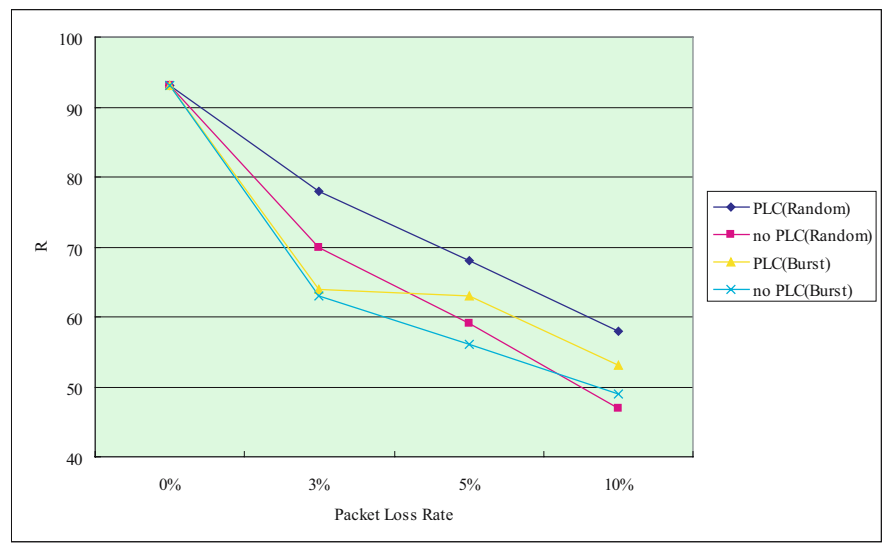

Fig. 11. Packet loss rate and $R$ rating

\section{Conclusions}

Voice-over-IP (VoIP) uses packetized transmission of speech over the Internet (IP network) and has been thought as one of the killer application of $\mathrm{BcN}$. In order for the Internet to realize a profit as traditional Public Switched Telephone Network (PSTN), it must provide competent quality of services for VoIP systems comparable to traditional PSTN systems. A successful end-to-end realization of IP telephony services presumes well-defined QoS measuring framework in the service provider's and customer's networks.

E-Model is regarded as prominent rating technology that can be applied most properly when estimate speech quality for VoIP service because it considering about data network characteristic such as loss, delay. This paper described a practical measuring framework based on E-model for end-to-end QoS of packet switched voice in an IP environment including Packet Loss Concealment (PLC) techniques and Network Time Protocol (NTP). We also investigated the effects of packet loss and delay jitter on speech quality in VoIP scenarios.

In addition to the block types defined RTCP XR, for VoIP monitoring, additional block types were defined in this paper by adhering to the RTCP XR framework.

\section{References}

1. William C. Hardy: VOIP Service Quality-Measuring and Evaluating Packet Switched-Voice, McGraw-Hill, (2003)

2. Victoria Fineberg: A Practical Architecture for Implementing End-to-End QoS in an IP Network, IEEE Communications Magazine, Jan. (2002) 122-130

3. T. Friedman, R. Caceres: RTP Control Protocol Extended Reports (RTCP XR), IETF RFC 3611, Nov. (2003) 
4. Jonathan Rosenberg, et. al.: SIP: Session Initiation Protocol, IETF RFC 3261, Jun. (2002)

5. Athina P. Markopoulou, Fouad A. Tobagi, and Mansour J. Karam: Assessing the Quality of Voice Communications Over Internet Backbones, IEEE/ACM Transaction on Networking, Vol. 11, No. 5, Oct. (2003) 747-760

6. Shengquan Wang, Zhibin Mai, Dong Xuan, and Wei Zhao: Design and Implementation of QoS-Provisioning System for Voice over IP, IEEE TRANSACTIONS ON PARALLEL AND DISTRIBUTED SYSTEMS, VOL. 17, NO. 3, MAR. (2006) 276-288

7. Shengquan Wang, Zhibin Mai, Walt Magnussen, Dong Xuan, and Wei Zhao: Implementation of QoS-Provisioning System for Voice over IP, IEEE Real-Time and Embedded Technology and Applications Symposium (RTAS02),

8. Definition of Categories of Speech Transmission Quality, ITU-T Recommendation G.109, (1999)

9. The E-Model, a computational model for use in transmission planing, ITU-T Recommendation G.107, (1998) 